\title{
Metal Liftoff Process using Solvent Soluble Resist by UV-NIL
}

\author{
Tomoki Nishino", Noriyoshi Fujii", Hiroto Miyake ${ }^{* *}$, Takao Yukawa ${ }^{* *}$, \\ Junji Sakamoto ${ }^{*}$, Ryosuke Suzuki ${ }^{*}$, Hiroaki Kawata ${ }^{*}$ and Yoshihiko Hirai \\ * Osaka Prefecture University \\ Department of Physics and Electronics Engineering, Graduate School of Engineering \\ 1-1 Gakuen-cho, Naka-ku, Sakai, Osaka, 599-8531,Japan \\ ${ }^{* *}$ Daisel Chemical Industries Ltd \\ Organic Chemical Products Company \\ 1239, Shinzaike, Aboshi-ku, Himeji, Hyogo, 671-1283,Japan
}

\begin{abstract}
Metal liftoff process has been demonstrated using solvent soluble UV cuable resin by UV nanoimprint lithography. Fine pattern is fabricated by UV nanoimprint using NIAC702 resist (Daisel Chemical), which is solved into Cyclohexanone. After removing residual layer by plasma etching, $\mathrm{Cr}$ is deposited by electron beam spattering and the resist is dissolved by Cyclohexanone. Using solvent soluble UV curable resin, $\mathrm{Cr}$ pattern is successfully transferred on the substrate by liftoff process.
\end{abstract}

Keywords: UV nanoimprint, metal, liftoff, plasmon, solvent,

\section{Introduction}

Nanoimprint lithography [1] is widely used for various industrial applications for fine pattern transfer in cost effective. Optical devices is one of the most promising applications for nanoimprint lithography because the transferred structures work as functional optical devices without any additional processes such as optical gratings, anti-reflection structures, wave plates and etc. We demonstrated anti-reflection structure using novel nanoimprint process [2], high aspect ratio wave plate [3], and optical grating using glass material [4]. Those applications were mostly using polymer materials.

On the other hand, plasmon devices [5] are rising up for new optical functional devices using nano structured materials such as nano metal dot array or 3 dimensional fine metal structures. To fabricate plasmon devices, electron beam lithography is usually applied, however it is not cost effective process because it takes long exposure time.

Nanoimprint lithography has been achieved to fabricate metal patterns by direct imprinting to soft metals [6,7], however residual metal layers are remained, which are not suitable for plasmon devices nor optical devices.

Fabrication of metal patterns using nanoimprint lithography was firstly successfully demonstrated by $\mathrm{S}$. Chou using PMMA by thermal nanoimprint process [1]. In thermal nanoimprint process, we have variety choices of resist materials and they are easily applied for metal liftoff process because polymers for thermal nanoimprint process are mostly solution dissolvable materials.

On the contrary, UV nanoimprint process [8] has high through put performance and is room temperature process. It is easy to handle the process, however UV curable resins are mostly insoluble after UV curing. To remove the cured resin, dry plasma etching is required, which is not applicable for liftoff process.

To dissolve the cured resin, multiple light exposure system has been proposed [9], however it is not suitable for conventional liftoff process. On the other hand, solvent soluble UV curable resin has been proposed [10], which is dissolved by organic solvent after UV curing.

In this paper, we newly propose metal liftoff process using solvent soluble resist by UV nanoimprint process. 


\section{Metal liftoff process}

Figure 1 shows the proposed process for metal liftoff. We use solvent soluble resist NIAC702 (Daisel Chemical), which is acrylate based radical polymerization UV curable resin. The resin is dissolved by Cyclohexanone and spin coated on $\mathrm{Si}$ substrate by $2000-3000 \mathrm{rpm}$. The resist is baked under $55^{\circ} \mathrm{C}$ for $1 \mathrm{~min}$. The initial thickness of the resist is approximately $200-400 \mathrm{~nm}$. Next, quartz template, which contains various patterns, is pressed on to the resist under $1.8 \mathrm{MPa}$ in pressure under vacuumed ambient and UV light is irradiated at $40 \mathrm{~mJ} / \mathrm{cm}^{2}$ in power for $60 \mathrm{~s}$. After releasing the template, the residual layer is removed by Inductive Coupled Plasma Reactive Ion Etching (ICP) using ULVAC NE-550 etching machine. The etching gas is $\mathrm{O}_{2}$ mixed with Ar.

Next, $\mathrm{Cr}$ is deposited by electron beam spatter using in-house equipment for around $50-200 \mathrm{~nm}$ in thickness. Finally, the resist is dissolved by Cyclohexanone and the $\mathrm{Cr}$ on the resist pattern is removed.

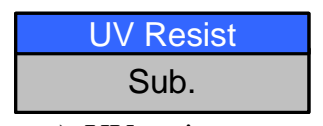

a) UV resist coat
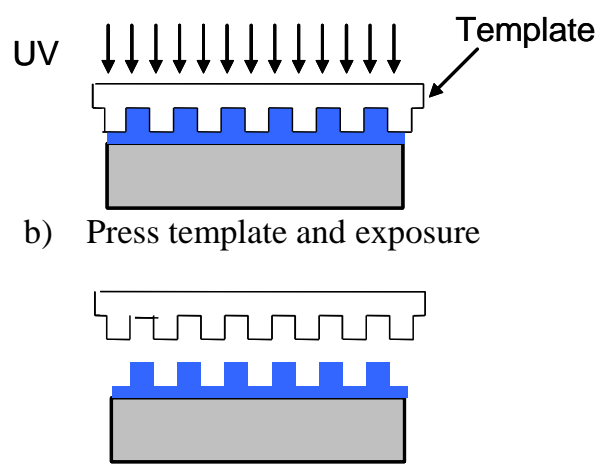

c) Release template

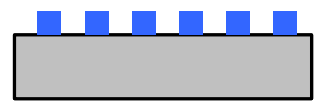

d) Remove residual layer

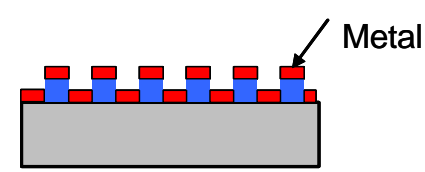

e) Metal deposition

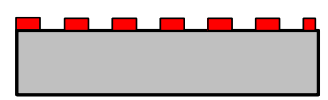

f) Dissolve resist and remove metal on the resist Figure 1. Schematic diagram of Metal liftoff process using solvent soluble resist by UV nanoimprint.

Figure 1. The proposed process for metal liftoff.

\section{Experimental result}

3.1 UV nanoimprint

Figure 2 shows typical experimental result for UV nanoimprint using NIAC 702 resist. Fine patterns are successfully fabricated on substrate without fatal defects. The residual layer thickness is around $200 \mathrm{~nm}$, which is removed before metal deposition by the following IPC etching.

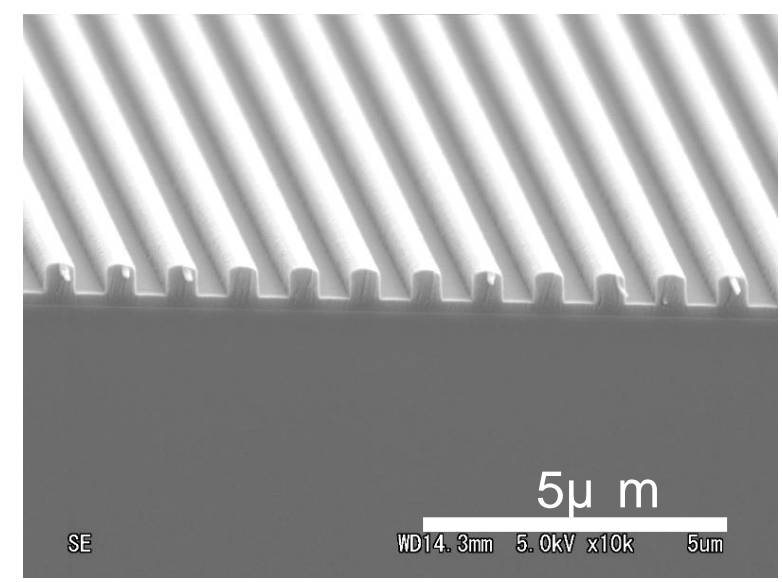

a) Low magnification view

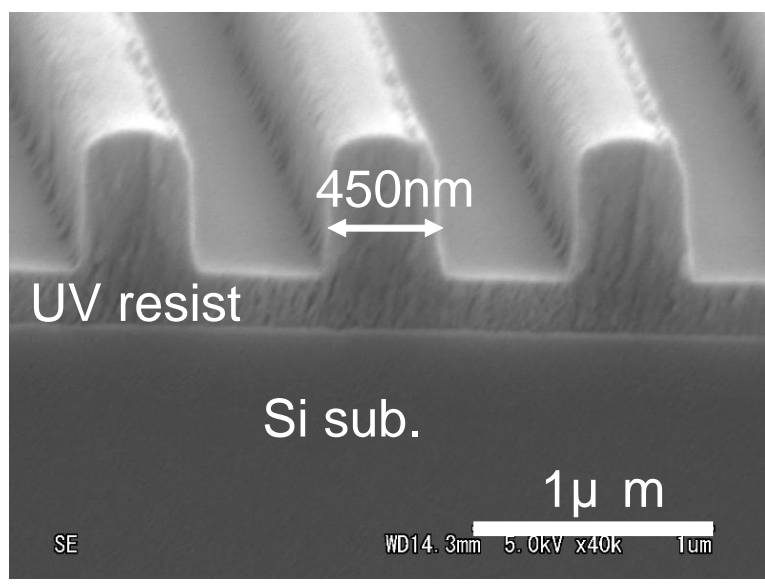

b) High magnification view

Figure 2. Cross Section view after UV nanoimprint.

\subsection{Residual layer removal}

Figure 3 shows typical result after removal of the residual resist layer by ICP etching. The residual layer is removed in anisotropically and the substrate is opened between the resist patterns. The pattern width is reduced in $40 \mathrm{~nm}$ during vertical etching over $200 \mathrm{~nm}$ in depth. The side wall of the pattern may be etched around $20 \mathrm{~nm}$ in lateral direction, which is fairly good anisotropic etching for polymer material. The cross section profile of the resist is not sharpened undercut profile and it is afraid that the side wall of the resist is covered with $\mathrm{Cr}$. 


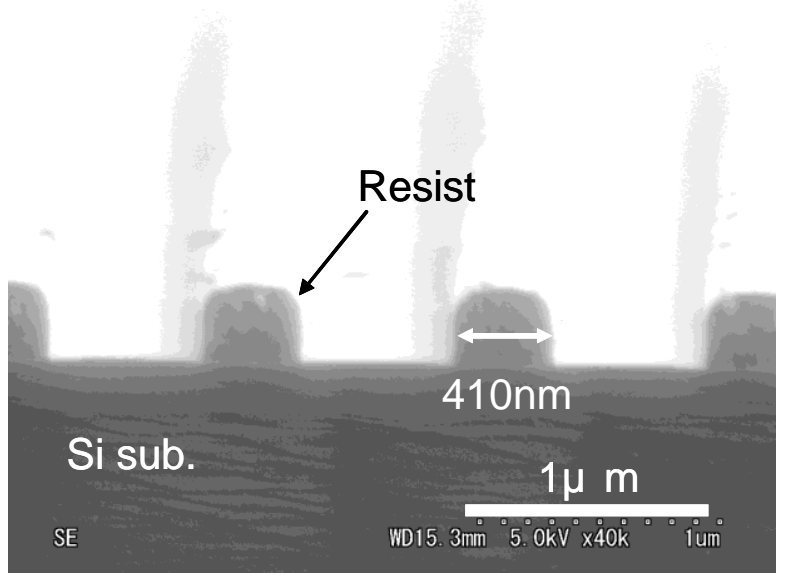

Figure 3. Cross Section view after residual layer removal by IPC etching

\subsection{Metal liftoff}

After deposition of $\mathrm{Cr}$ layer, the sample is dipped into Cyclohexanone for $15 \mathrm{~min}$ to remove the resist. Figure 4 shows typical result after dipping in Cyclohexanone.

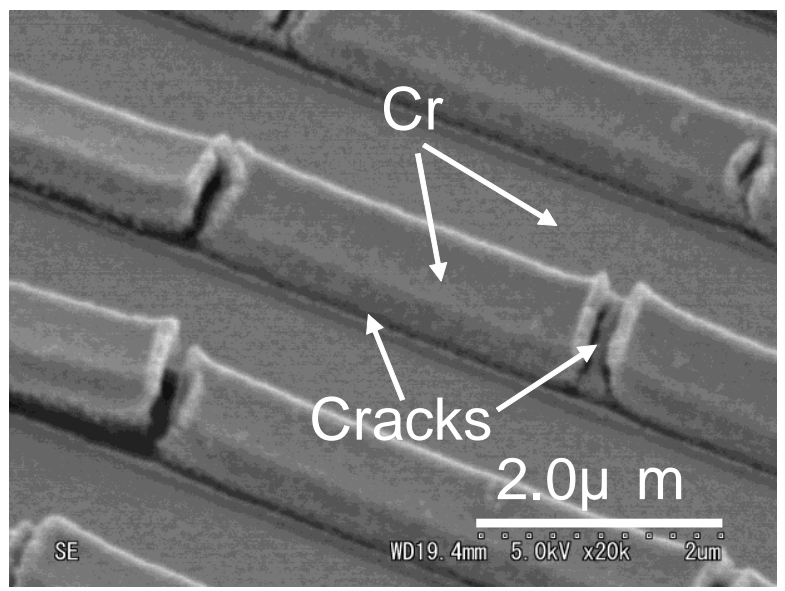

a) Line patterns

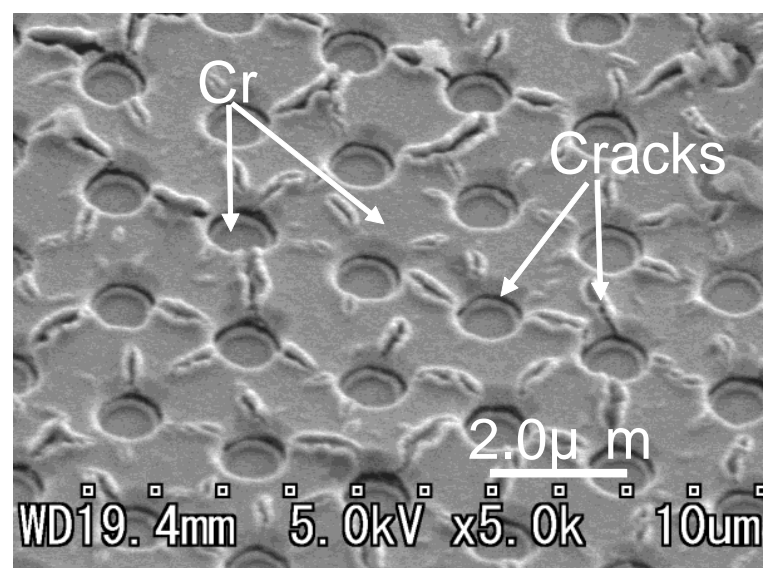

b) Dot array patterns

Figure 4. Bird's eye view after Cyclohexanone dipping. (15 min dipping)
The Cr layer on the rest does not removed with the resist and still remained, however some cracks are observed on the $\mathrm{Cr}$ layer and also pattern edges.

To remove the underneath resist layer, extra dipping under ultrasonic vibration is carried out in Cyclohexanone for $15 \mathrm{~min}$.

Figures 5 and 6 show the results of Cr liftoff after extra dissolving under ultrasonic vibration in solvent.

Fine $\mathrm{Cr}$ lines and dot array with $600 \mathrm{~nm}$ in feature size are successfully transferred by liftoff process using solvent soluble UV resist, respectively. Under ultrasonic dipping, the solvent flow into the resist through the cracks and dissolved. As a result the Cr layers on the resist are successfully removed

As demonstrated above, $\mathrm{Cr}$ patterns are transferred by UV nanoimprint process using solvent soluble resist.

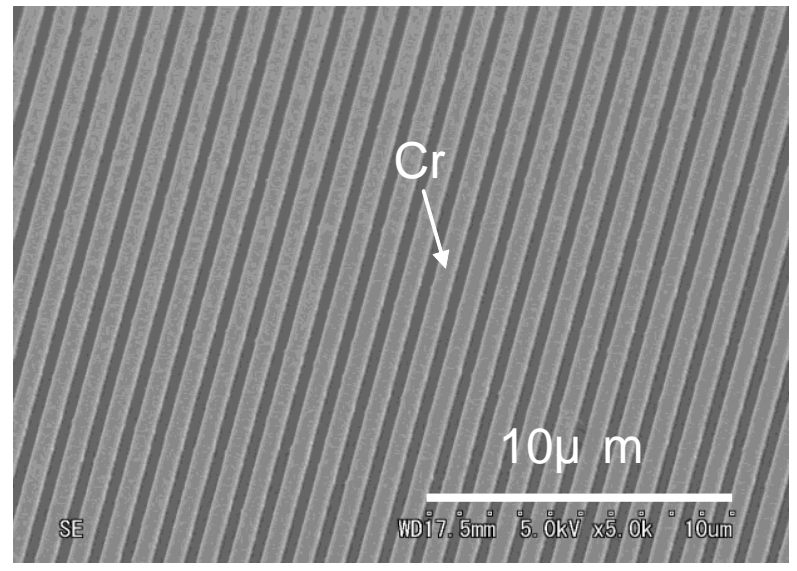

a) Low magnification view

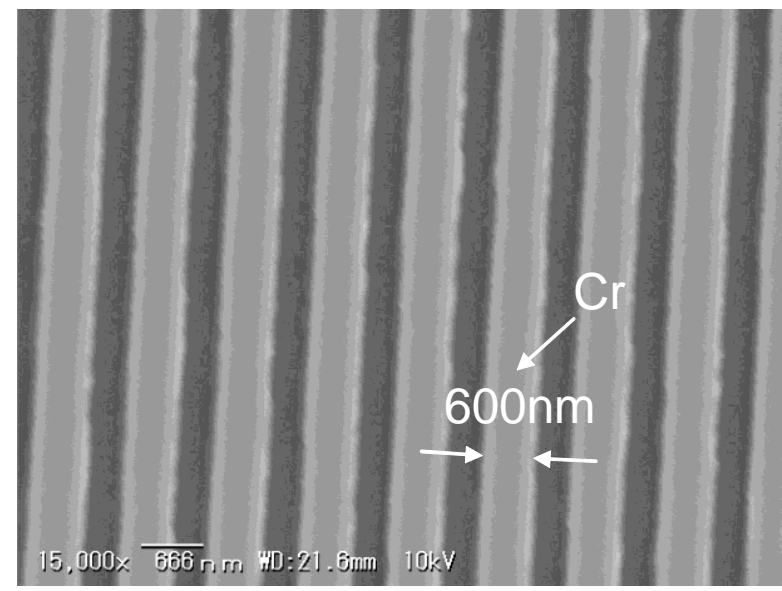

b) High magnification view

Figure 5 Transferred $\mathrm{Cr}$ lines by liftoff process 


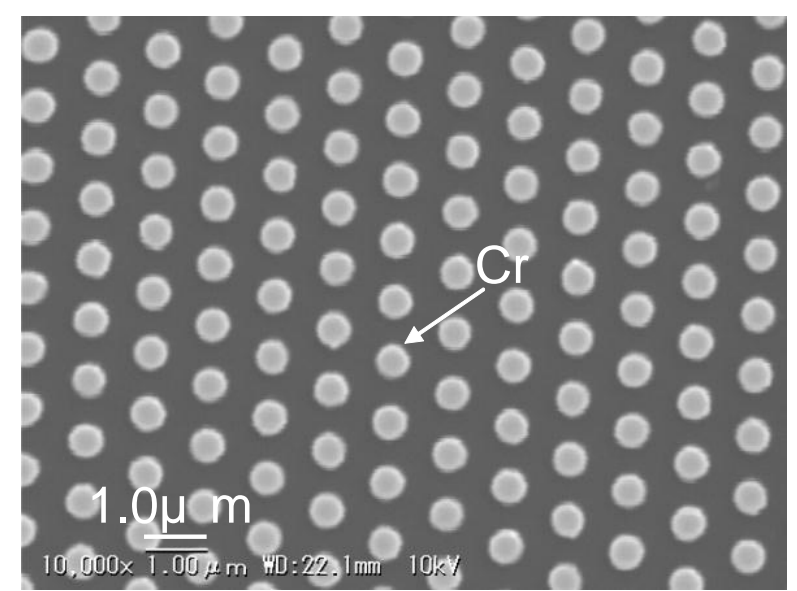

a) Low magnification view

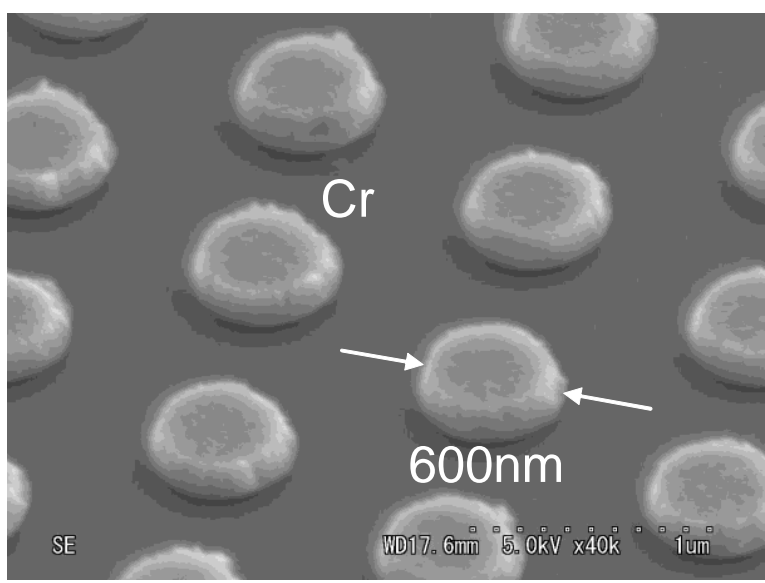

b) High magnification view

Figure 6. Transferred $\mathrm{Cr}$ dot array by liftoff process

\section{Conclusion}

Metal liftoff process using solvent soluble UV curable resin is newly proposed by UV nanoimprint process. Fine $\mathrm{Cr}$ line pattern and dot array pattern are successfully demonstrated.

We believe liftoff process in combination with UV nanoimprint lithography is promising way to fabricate metal nano patterns for plasmon devices in cost effectively.

\section{References}

1. S. Chou, P. Krauss, and P. Renstrom, Appl. Phys. Lett. 67, (1995) 3114.

2. K. Sogo, M. Nakajima and Y. Hirai, $J$. Photopolym. Sci. Technol., 19, (2006) 647.

3. T. Yoshikawa, T. Konishi, M. Nakajima, H.Kikuta, H. Kawata, and Y. Hirai, J. Vac. Sci. Technol. B 23(2005) 2939.

4. Y. Hiraia, K. Kanakugi, T. Yamaguchi, K. Yao, S. Kitagawa, Y. Tanaka, Microelectronic Engineering 67-68 (2003) 237.

5. for example, H. Atwater, Scientific American (April 2007) 56.

6. S. Pang, T. Tamamura, N. Makao, A. Ozawa, and H. Masuda, J. Vac. Sci. Technol.B, 16 (1998) 1145.

7. Y. Hirai, T. Ushiro, T. Kanakugi, T. Matsuura, Proc. of SPIE 5220, (2003) 74.

8. M. Colburn, S. Johnson, M. Stewart, S. Damle, T. Bailey, B. Choi, M. Wedlake, T. Michaelson, S.V. Sreenivasan, J. Ekerdt, and C.G. Willson: Proc. of SPIE 3676 (1999) 378.

9. D. Matsukawa, H. Wakayama, K. Mitsukura, H. Okamura, Y. Hirai and M. Shirai, J. Mater. Chem. 19 (2009) 4085.

10. T. Yukawa, S. Iyoshi and H. Miyake, Proc. Jpn. Soc. Appl. Phys. annual meeting (2009, Tukuba) 30a-K- 9, in Japanese. 